\section{Off-Label-Use: Der Fall Methylphenidat}

Jürgen Fritze \& Max Schmauß

für den Vorstand der Deutschen Gesellschaft für Psychiatrie, Psychotherapie und Nervenheilkunde (DGPPN)

D ie Probleme der Ablehnung des Off-Label-Use von NeuroPsychopharmaka durch die Krankenkassen vor dem Hintergrund des Urteils des Bundessozialgerichts wurden inzwischen mehrfach diskutiert (Nervenarzt 2002; 73: 796-799 und 1125-1127). Die Deutsche Gesellschaft für Psychiatrie, Psychotherapie und Naturheilkunde (DGPPN) hat einen Katalog von Indikationen, in denen Off-Label-Use von Psychopharmaka als dem Stand der Wissenschaft entsprechend anzusehen ist (in Psycho 2002; 28: 431-439 und bei www. dgppn.de), präsentiert.

Der Einsatz des Raucher-Entwöhnungsmittels Bupropion und

\section{Verordnung (Mio. DDD) von Methylphenidat zu Lasten der GKV (Arzneiverordnungsreport 2002)}

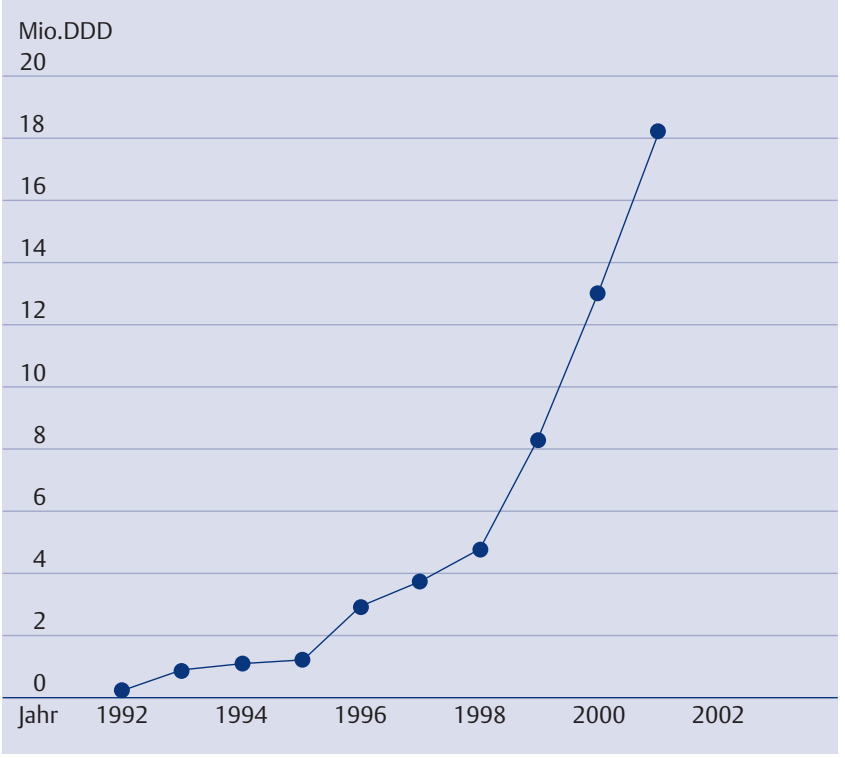

renzen unter Beteiligung auch von Angehörigenverbänden initiiert. Implizites Ziel dieser Initiative ist, die Verordnung von Methylphenidat an bestimmte Voraussetzungen zur Strukturqualität aufseiten der Verordner zu binden.

Laut Fachinformation „hat Methylphenidat bei nicht bestimmungsgemäßem Gebrauch ein stark ausgeprägtes psychisches Abhängigkeitspotenzial. Bei bestimmungsgemäßem Gebrauch in den zugelassenen Anwendungsgebieten ist die Abhängigkeitsgefahr gering bzw. praktisch nicht vorhanden. Missbrauch und Abhängigkeit scheinen auch bei Jugendlichen und Erwachsenen, die als Kinder Methylphenidat zur Therapie hyperkinetischer Verhaltensstörungen erhalten haben, nicht vorzukommen. Auch bei Erwachsenen mit Narkolepsie spielt das Abhängigkeitsrisiko praktisch keine Rolle."

Die Verordnung ist mit einem vertretbaren Nutzen-Risiko-Verhältnis also nur dann vertretbar, wenn die Indikation zutrifft. Deshalb macht die Initiative des BMGS grundsätzlich Sinn. Allerdings stellt sich die Frage, ob die Bindung an Voraussetzungen zur Strukturqualität oder nicht eher Maßnahmen zur Sicherung der Ergebnisqualität sachgerecht sind. Wird Methylphenidat außerhalb dieser Indikationen (d.h. auch außerhalb eines therapeutischen Gesamtkonzeptes) verordnet, also „off-label“, so kann man sich eines vertretbaren NutzenRisiko-Verhältnisses nicht mehr sicher sein.

Methylphenidat ist zugelassen zur Anwendung bei „hyperkinetischen Verhaltensstörungen bei Kindern im Rahmen einer therapeutischen Gesamtstrategie" und bei „zwanghaften Schlafanfällen während des Tages (Narkolepsie) im Rahmen einer therapeutischen Gesamtstrategie“. In der Vorschlagsliste verordnungsfähiger Arzneimittel (sog. Positivliste) des Institutes für die Arzneimittelverordnung in der gesetzlichen Krankenversicherung (GKV) des BMGS ist die Indikation bei Verordnung zu Lasten der GKV eingeschränkt: „nur zur Behandlung des hyperkinetischen Syn- 
droms". In jedem Fall handelt es sich bei Verordnung an Erwachsene (jenseits des 18. Lebensjahres) zum Zweck der Behandlung einer Aufmerksamkeitsstörung (ADS/ADHS) um Off-Label-Use, da sich der Indikationsanspruch auf das Kindesalter beschränkt.

Mit ausdrücklichem Hinweis auf das Urteil des Bundessozialgerichts (BSG) vom März 2002 erwarten zumindest einzelne Medizinische Dienste der Krankenkassen (MDK) bei Verordnung an Erwachsene zur Behandlung einer Aufmerksamkeitsstörung detaillierte Darlegungen zu folgenden Punkten:

„Anamnestische Angaben zu Beginn und Dauer der Erkrankung; aktueller psychopathologischer Befund, aus dem sich die Schwere der Erkrankung im Einzelfall ableiten lässt; Angaben zu Dauer der Symptomatik und Ausprägungsgrad in den letzten zwei bis drei Jahren; konkrete Einzelfall-bezogene Angaben zu den Auswirkungen der Erkrankung auf Befinden, Partnerbeziehung, Beruf und soziales Umfeld als Gradmesser der Auswirkungen der Störung und zur Abschätzung der Beeinträchtigung der Lebensqualität; Prognose hinsichtlich des weiteren Krankheitsverlaufes und seiner körperlichen und/oder psychosozialen Auswirkungen; Darlegung der bisherigen medikamentösen Therapien nach Dosis, Applikationsdauer, therapeutischen und unerwünschten Wirkungen; Darlegung der bisherigen Psychotherapien nach Art, Dauer, therapeutischen und unerwünschten Wirkungen; Mitteilung, ob die medikamentöse Behandlung mit der beantragten Wirksubstanz in ein multimodales Konzept eingebunden werden soll und worin dieses gegebenenfalls besteht."

Es gehe darum, „die nachhaltige Beeinträchtigung der Lebensqualität auf Dauer für den Einzelfall zu belegen und für den Einzelfall nachzuweisen, dass andere Therapiemöglichkeiten (wie z.B. kognitive und Verhaltenstherapie) nicht ausreichend geholfen haben oder nicht ohne gleichzeitige Pharmakotherapie eingesetzt werden können.“

Der MDK fordert also ein aus- führliches Gutachten in jedem Einzelfall, seltsamerweise aber ohne dabei auf die dritte vom BSG formulierte Bedingung einzugehen, inwiefern „auf Grund der Datenlage die begründete Aussicht besteht, dass mit dem betreffenden Präparat ein Behandlungserfolg (kurativ oder palliativ) zu erzielen ist." Zumindest dieser MDK scheint also davon auszugehen, dass die Wirksamkeit von Methylphenidat bei der residualen Aufmerksamkeitsstörung (ADS/ ADHS) des Erwachsenen hinreichend belegt ist. Damit räumt dieser MDK aber ein, dass es sich eigentlich nicht um Off-Label-Use handelt. Mit seinen Forderungen setzt der MDK nur die vom BMGS mit seiner o.g. Initiative erwarteten Qualitätsanforderungen um, dies allerdings im Vorgriff auf den vom BMGS angestrebten Konsens.

Welche Auswirkungen die Forderung eines ausführlichen, d.h. mit erheblichem Aufwand verbundenen Gutachtens in jedem Einzelfall auf die ohnehin bei Erwachsenen mit ADS/ADHS bestehende Unterversorgung haben wird, soll der Fantasie des Lesers überlassen bleiben. Dasselbe gilt für den im Vergleich zur o.g. Initiative „vorauseilenden Gehorsam" dieses MDK. Von Interesse ist aber zu prüfen, inwiefern die Forderungen im Sinne von Off-LabelUse sachgerecht sind.

Die Zulassung eines Indikationsanspruchs durch die Behörde (hier Bundesinstitut für Arzneimittel und Medizinprodukte, BfArM) setzt den Beleg von Wirksamkeit, Verträglichkeit und Unbedenklichkeit in der betroffenen Population - unter Würdigung des Nutzen-Risiko-Verhältnisses - voraus. Die Zulassung von Methylphenidat beschränkt sich auf ADS/ADHS im Kindesalter bzw. ohne Altersbindung die Narkolepsie, jeweils im Rahmen eines therapeutischen Gesamtkonzepts. Dabei kann eine Zulassung nur für jenen Indikationsanspruch ausgesprochen werden, der vom Hersteller beantragt wurde.

Die Altersbindung für die Indikation ADS/ADHS ergibt sich daraus, dass zurzeit ihrer Beantragung die ADS/ADHS des Erwachsenen weitgehend unerkannt war und entsprechend keine Studien bei Erwachse- nen vorliegen konnten. Hieran hat sich wenig geändert. Die Übersicht von Krause \& Ryffel-Rawak (2) konnte nur fünf randomisierte Studien auflisten, die alle an methodischen Mängeln (u.a. geringe Fallzahlen, Crossover-Design) leiden. Seither sind drei weitere Studien, ebenfalls mit methodischen Mängeln, publiziert worden. Die Studie von Schubiner et al. (4) untersuchte 48 kokainabhängige ADS/ADHS-Fälle und ist damit kaum generalisierbar. Die Studie von Dorrego et al. (1) verglich Methylphenidat mit Lithium im Crossover-Design (und fand vergleichbare Wirkungen). Die Studie von Kuperman et al. (3) verglich Methylphenidat mit Bupropion und Plazebo bei 30 Patienten und fand nur einen Trend $(p=0,14)$ zu Gunsten der aktiven Behandlungen; dieses Scheitern ist möglicherweise der zu geringen Fallzahl zuzuschreiben.

Trotz dieser Mängel hat der o.g. MDK recht, an der Wirksamkeit von Methylphenidat bei der ADS/ADHS des Erwachsenen nicht zu zweifeln: Grundsätzlich wird zulassungsrechtlich davon ausgegangen, dass bei Kindern und Erwachsenen bei identischer Indikation Wirksamkeit gegeben ist. Altersgruppen-spezifischer Wirksamkeitsnachweis ist zu verlangen, wenn es sich um Indikationen handelt, die nur altersgebunden vorkommen. In jedem Fall sind aber die altersgebundenen Spezifika der Pharmakokinetik und damit zusammenhängend der Dosierung gezielt zu untersuchen. Wenn also was unzweifelhaft ist - die Wirksamkeit von Methylphenidat bei Kindern mit ADS/ADHS belegt ist, so darf die Wirksamkeit bei Erwachsenen mit ADS/ADHS angenommen werden, zumindest vor dem Hintergrund der auch für Erwachsene vorliegenden Wirksamkeitsstudien.

Dies gilt nicht notwendigerweise für Verträglichkeit und Unbedenklichkeit. Es ist nicht auszuschließen, dass sich insbesondere das Abhängigkeitsrisiko bei Erwachsenen anders als bei Kindern darstellen könnte. Hier wäre aber nicht plausibel zu erwarten, dass sich das Risiko einer Abhängigkeit zwischen den Indikationen ADS/ADHS des Erwachsenen und Narkolepsie des 
Erwachsenen unterscheidet. Ausdrücklich ist Methylphenidat für die Anwendung bei der Narkolepsie des Erwachsenen zugelassen, d.h. im Analogieschluss darf das (laut mit der Zulassungsbehörde abgestimmter Fachinformation bei bestimmungsgemäßem Gebrauch vernachlässigbare) Abhängigkeitsrisiko auch bei Anwendung in der Indikation ADS/ADHS des Erwachsenen als vertretbar angesehen werden.

Zum bestimmungsgemäßen (zugelassenen) Gebrauch gehören nicht nur Indikation, Alters- und ggf. Geschlechtsspezifikation, sondern Dosis und Behandlungsdauer. Bleibt also, dass formelle, methodisch adäquate Dosisfindungsstudien für Methylphenidat im Erwachsenenalter und Daten zur Behandlungsdauer fehlen. Dies gilt - aus historischen Gründen - für das Kindesalter gleichermaßen. Dabei ist zu berücksichtigen, dass (laut Fachinformation) „die Dosis den Erfordernissen des Einzelfalls angepasst werden muss. Die Therapie mit Methylphenidat sollte mit einer niedrigen Dosis begonnen und in kleinen Stufen bis zum Erreichen einer verträglichen und genügend wirksamen Dosis gesteigert werden. Hierbei gilt der Grundsatz, die Dosis so klein wie möglich zu halten." Die Notwendigkeit von Dosisfindungsstudien relativiert sich also angesichts der Notwendigkeit individueller Dosierung. Also kann das Fehlen von Dosisfindungsstudien nicht als Indikator angesehen werden, die Anwendung von Methylphenidat bei ADS/ADHS des Erwachsenen stelle Off-Label-Use dar.

Folglich erfüllt die Anwendung von Methylphenidat bei ADS/ADHS des Erwachsenen nicht das Merkmal, mit dem das BSG eine Leistungspflicht der Krankenkasse bei Off-LabelGebrauch ausschließt, nämlich „weil für das neue Anwendungsgebiet weder die Wirksamkeit noch etwaige Risiken des Arzneimittels in dem nach dem Arzneimittelgesetz vorgeschriebenen Zulassungsverfahren geprüft worden sind“. Folglich kann der MDK keine umfänglichen Gutachten in jedem Einzelfall fordern - es sei denn, die vom BMGS initiierte Konsensuskonferenz würde solche Forderungen erheben.

\section{Literatur}

1. Dorrego MF, Canevaro L, Kuzis G, Sabe L, Starkstein SE. A randomized, double blind, crossover study of methylphenidate and lithium in adults with attention deficit/hyperactivity disorder: Preliminary findings. J Neuropsychiatry Clin Neurosci 2002; 14: 289-295

2. Krause J, Ryffel Rawak D. Therapie der Aufmerksamkeitsdefizit/Hyperaktivitätsstörung im Erwachsenenalter. Psycho 2000; 26: 209-223 3. Kuperman S, Perry PJ, Gaffney GR, Lund BC, Bever Stille KA, Arndt S, Holman TL, Moser DJ, Paulsen JS. Bupropion SR vs. methylphenidate vs. placebo for attention deficit hyperactivity disorder in adults. Ann Clin Psychiatry 2001; $13: 129-134$

4. Schubiner H, Saules KK, Arfken CL, Johanson C E, Schuster CR, Lockhart N, Edwards A, Donlin J, Pihlgren E. Double blind placebo controlled trial of methylphenidate in the treatment of adult ADHD patients with comorbid cocaine dependence. Exp Clin Psychopharmacology 2002; 10 : 286-294

5. The European Agency for the Evaluation of Medicinal Products (EMEA). Note for guidance on clinical investigation of medicinal products in children, 1997

\section{Korrespondenzadresse:}

Prof. Dr. med. J. Fritze

Asternweg 65

50259 Pulheim
Georg Thieme Verlag

Rüdigerstraße 14

70469 Stuttgart

Telefon (0711) 89 31-0

Telefax (0711) 89 31-322

Verantwortliche Schriftleitung:

Markus Gastpar, Essen

Jürgen Fritze, Pulheim

Erweiterte Schriftleitung:

Günther Faust, Mainz

Manfred Fichter, Prien am Chiemsee

Andreas Hufnagel, Essen

Joachim Morgner, Dresden

Götz-Erik Trott, Aschaffenburg

Wissenschaftlicher Beirat:

Thomas Becker, Leipzig; Horst Berzewski, Berlin; Lothar Blaha, Deggendorf;

Hans-Dieter Brenner, Bern; Pasquale

Calabrese, Bochum; Klemens Dieckhöfer, Bonn; Tilman Elliger, Köln; Volker Faust, Ravensburg; Franz loseph Freisleder, München; Michael Geyer, Leipzig; Iver Hand, Hamburg; Gerhard Harrer Iver Hand, Hamburg; Gerhard Harrer,

Paul Hoff, Aachen; Siegfried Kaumeier, Merzig; Roger Kirchner, Cottbus; Gerd Merzig; Roger Kirchner, Cottbus; Gerd Laux, Wasserburg/München; Helmut furt am Main; Dieter Naber, Hamburg; Hartmut Radebold, Kassel; Helmut Remschmidt, Marburg; Peter Riederer, Würzburg; Eckardt Rüther, Göttingen; örn Peter Sieb, München; Wolfgang Weig

Merzig: Roland Wörz, Bad Schönborn; Manfred Wolfersdorf, Bayreuth

Bestellungen: Über den Buchhandel oder direkt beim Verlag

Bezugsbedingungen:

Einzelheft $€ 9,-+$ Porto.

lahresabonnement $€ 69$,

inkl. MwSt. und Porto.

Für Medizinstudenten

gegen Nachweis und Bankeinzug

$€ 45$,- inkl. MwSt. und Porto.

Ausland zuzüglich Versandkosten

(cash with order) $€ 15,80$ (Europa)

bzw. $€ 44,90$ (Airlift)

Der Abonnementpreis umfasst $12 \mathrm{Ka}$ lendermonate (Mindestlaufzeit). Abonnements laufen weiter, wenn nich 3 Monate vor lahresende eine Abbestellung beim Verlag vorliegt.

Bei Nichtbelieferung im Falle höherer Gewalt, bei Störungen des Betriebsfriedens, Arbeitskampf (Streik, Aussperrung) bestehen keine Ansprüche gegen den Verlag.

Verlag und Copyright:

(c) 2003 by Georg Thieme Verlag,

Rüdigerstraße 14, 70469 Stuttgart.

Mit dem Abdruck des Beitrages erwirbt der Verlag das alleinige und ausschließliche Recht für die Veröffentlichung in sämtlichen Publikumsmedien sowie Übersetzungen in fremde Sprachen. Nachdruck, fotomechanische Wiedergabe und Speicherung in den Datenverarbeitungsanlagen, auch auszugsweise, nur nach schriftlicher Genehmigung des Verlages. Die Abbildungen in den Verlages. Die Abbildungen in den Beiträgen stammen, wenn nicht anders vermerkt, von den jeweiligen Autoren. Fü Inhalt, Vorschau und Titelseite werden Abbildungen aus den beschriebenen $\mathrm{Ar}$ beiten verwendet, anderenfalls ist die Herkunft ebenfalls im Inhaltsverzeichnis deklariert.

\section{Hinweis für Autoren:}

Zur Veröffentlichung in der Zeitschrift angenommen werden unveröffentlichte Originalarbeiten, Übersichtsarbeiten und Kasuistiken, die nicht auch gleichzeitig an anderer Stelle zur Veröffentlichung eingereicht sein dürfen. Richtlinien zur Abfassung des Manuskriptes können bei der Redaktion angefordert werden.

Die eingereichten Arbeiten für Editorial, Schwerpunkt/Brennpunkt und Übersicht/Originar sicht/Originalarbeit werden einem pee review der schriftleitung unterzogen. Der Inhalt der ubrigen Rubriken und de Supplements liegt in der Verantwortung der Redaktion.

\section{Redaktionsleitung: \\ Günther Buck \\ Telefon (0711) 89 31-440 \\ Chefredaktion: \\ Katrin Wolf \\ Telefon (0711) 89 31-179 \\ Katrin.Wolf@thieme.de \\ Redaktionsassistenz: \\ Sabine Bischoff \\ Telefon (0711) 89 31-551 \\ Telefax (0711) 8931-322 \\ Internet-Adresse:}

http://www.thieme.de

Verantwortlich für den Anzeigenteil:

pharmedia, Anzeigen-

und Verlagsservice $\mathrm{GmbH}$

Anzeigenleitung:

Greta Weller

Telefon (0711) 8931-304

Greta.Weller@pharmedia.de

Zur Zeit gilt Anzeigenpreisliste

1.10.2002

Zeitschriftenvertrieb:

Telefon (0711) 89 31-321

Herstellung:

Karl-Heinz Zobel

Telefon (0711) 89 31-441

Satz, Grafik, Layout:

Wolfgang Eckl, Andrea Stiller,

Karl-Heinz Zobel

Druck und Verarbeitung:

W. Kohlhammer Druckerei GmbH + Co 70329 Stuttgart

Bankverbindung

Deutsche Bank Stuttgart

Konto-Nr. 1420 017, BLZ 60070070

Landesbank Baden-Württemberg

Konto-Nr. 2055 723, BLZ 60050101

Postgiro Stuttgart

Konto-Nr. 45 000-705, BLZ 60010070

Erscheinungsweise: monatlich

Regularly listed in EMBASE

Hinweis:

Wie jede Wissenschaft ist die Medizin ständigen Entwicklungen unterworfen. Forschung und klinische Erfahrung erweitern unsere Erkenntnis, insbesondere was Behandlung, medikamentöse Therapie sowie Diagnostik (Laborwerte etc.) anbelangt. Soweit in dieser Zeitschrift Dosierungen Applikationen oder scher Laborf der Leser zur darauforen, Herausgeber und Verlag große Sorgfalt darauf verwandt haben, das diese Angaben dem Wissensstand be Fertigstellung entsprechen. Für Angaben über Dosierungsanweisungen, Applikationsformen und Laborwerte kann vom Verlag jedoch keine Gewähr übernommen werden. Jeder Benutzer ist angehalten, durch sorgfältige Prüfung der Beipackzettel der verwendeten PräpaBeipackzetel der vor rate und ggf. nach Konsultation eine gezialisten festzustellen, ob die dort gegebene Empfehlung für Dosierunge oder die Beachtung von Kontraindikatio nen gegenüber der Angabe in dieser Zeitschrift abweicht. Eine solche Prüfung ist besonders wichtig bei selten verwendeten Präparaten oder solchen, die neu auf den Markt gebracht worden sind. Jede Dosierung oder Applikation erfolgt auf eigene Gefahr des Benutzers. Laborwerte müssen immer auf Ihre Plau sibilität silian gig vom jeweiligen Testgerät bzw. Test kit. Autoren und Verlag appellieren an jeden Benutzer, ihm auffallende Unge-
nauigkeiten dem Verlag mitzuteilen. 